\title{
Structural mimicry between SLA/LP and Rickettsia surface antigens as a driver of autoimmune hepatitis: insights from an in silico study
}

\author{
Alessandro Paiardini and Stefano Pascarella
}

\author{
* Correspondence: alessandro. \\ paiardini@uniroma1.it \\ Dipartimento di Scienze \\ Biochimiche "A. Rossi Fanelli", \\ Sapienza - Università di Roma, \\ 00185, Roma, Italy
}

\begin{abstract}
Background: Autoimmune hepatitis $(\mathrm{AlH})$ is a chronic, progressive liver disease, characterized by continuing hepatocellular inflammation and necrosis. A subgroup of AlH patients presents specific autoantibodies to soluble liver antigen/liver-pancreas (SLA/LP) protein, which is regarded as a highly specific diagnostic marker. Autoantigenic SLA/LP peptides are targeted by $C D 4^{+} T$ cells, and restricted by the allele HLA-DRB1*03:01, which confers disease susceptibility in Europeans and Americans. A positively charged residue at position 71 has been indicated as critical for AlH susceptibility in all of the HLA alleles identified to date. Though the exact molecular mechanisms underlying pathogenesis of AlH are not clear, molecular mimicry between SLA/LP and viral/bacterial antigens has been invoked.

Methods: The immunodominant region of SLA/LP was used as query in databank searches to identify statistically significant similarities with viral/bacterial peptides. Homology modeling and docking was used to investigate the potential interaction of HLA-DRB1*03:01 with the identified peptides. By molecular mechanics means, the interactions and energy of binding at the HLA binding site was also scrutinized.

Results: A statistically significant structural similarity between the immunodominant regions of SLA/LP and a region of the surface antigen PS 120 from Rickettsia spp. has been detected. The interaction of the SLA/LP autoepitope and the corresponding Rickettsia sequence with the allele HLA-DRB1*03:01 has been simulated. The obtained results predict for both peptides a similar binding mode and affinity to HLA-DRB1*03:01. A "hot spot" of interaction between HLA-DRB1*03:01 and PS 120 is located at the P4 binding pocket, and is represented by a salt bridge involving Lys at position 71 of the HLA protein, and Glu 795 of PS120 peptide.

Conclusions: These findings strongly support the notion that a molecular mimicry mechanism can trigger AlH onset. CD4 ${ }^{+}$T cells recognizing peptides of SLA/LP could indeed cross-react with foreign Rickettsia spp. antigens. Finally, the same analysis suggests a molecular explanation for the importance of position 71 in conferring the susceptibility of the allele HLA-DRB1*03:01 to AlH. The lack of a positive charge at such position could prevent HLA alleles from binding the foreign peptides and triggering the molecular mimicry event.
\end{abstract}

Keywords: Autoimmune hepatitis, Molecular mimicry, Rickettsia, SLA/LP, Peptide conformation, PLP-dependent enzymes, HLA-DRB1*03:01 


\section{Background}

Autoimmune hepatitis $(\mathrm{AIH})$ is a chronic, progressive liver disease, characterized by continuing hepatocellular inflammation and necrosis. Autoantibodies to several antigens represent a serological feature of $\mathrm{AIH}$, though most of them are not specific for the disease [1]. In contrast, autoantibodies to soluble liver antigen and to liver-pancreas (SLA/LP) have been described as disease specific, suggesting their potential involvement in the pathogenesis of AIH, at least in the subgroup of patients presenting SLA/LP autoantibodies (about $20 \%$ of AIH cases) $[2,3]$. Expression, cloning and absorption experiments identified a protein with homology to a putative UGA suppressor tRNA-associated protein, as the sole target antigen of SLA/LP autoantibodies [4]. This protein had been previously identified as it coprecipitated with $\mathrm{tRNA}^{\mathrm{Sec}}$, when mammalian cell extracts were treated with serum from patients with AIH [5]. Subsequent in vivo and in vitro results therefore identified SLA/LP as $O$-phosphoserine (Sep)-tRNA:selenocysteine (Sec)-tRNA synthase (SepSecS, according to the Nomenclature Commission of the Human Genome Organization). SLA/LP belongs to the superfamily of pyridoxal 5 '-phosphate (PLP) dependent enzymes of "fold type I" [6,7], sharing the same fold and high structural similarity with other members of this group [8,9], and catalyzing the last step of Sec synthesis, i.e., the conversion of Sep-tRNA ${ }^{\text {Sec }}$ to Sec-tRNA ${ }^{\text {Sec }}[10]$. By studying carboxy-terminally truncated SepSecS, Wies et al. [4] identified an immunodominant region that is specifically recognized by SLA/LP autoantibodies, and which is located between residues 450-490.

Whereas autoantibodies represent a serological feature of $\mathrm{AIH}$, intrahepatic lymphocytic infiltrates are the histologic hallmark of $\mathrm{AIH}$, and are regarded as the primary factor for disease pathogenesis [11]. Indeed, intrahepatic $\mathrm{CD} 4^{+} \mathrm{T}$ cells recognize self-antigens in the context of the alleles HLA-DRB1*03:01 and HLA-DRB1*0401, which represent the principal AIH susceptibility alleles among Europeans and Americans, and in the context of alleles HLA-DRB1*04:05 and HLA-DRB1*04:04, which are instead linked to AIH susceptibility in Japan, Argentina and Mexico [12,13]. In particular, a positively charged residue at position 71 in the context of the region $67-72$ of the DR $\beta$ polypeptide corresponding to the LLEQ $[K / R] R$ motif, which is shared among the above mentioned HLA alleles, has been indicated as critical for AIH susceptibility [14]. Recently, HLA-DRB1*03:01 transgenic mice were immunized with SLA/LP, with the aim of identifying auto-antigenic SLA/LP peptides that are targeted by $\mathrm{CD} 4^{+} \mathrm{T}$ cells, and restricted by the disease susceptibility gene HLA-DRB1 "03:01 [15]. Interestingly, the observation that the C-terminal region of SLA/LP spanning residues 452-465 (sequence NRLDRCLKAVRKER), which was identified as the optimal $\mathrm{CD}^{+} \mathrm{T}$ cell epitope, overlaps with the SLA/LP sequence that is recognized by antibodies of patients with AIH suggests that the C-terminal region of SLA/LP is not only targeted by humoral, but also by cellular immune responses $[4,15]$.

To date, the exact molecular mechanisms that initiate and maintain the production of autoantibodies in AIH are not clear, though the remarkable uniformity in epitope recognition shown by SLA/LP autoantibodies suggests a common mechanism [16]. Molecular mimicry between self-antigens and antigens from infectious agents has been suggested as a mechanism for the generation of autoantibodies [17]. Therefore, it has been hypothesised that autoimmunity to the SLA/LP protein might be driven by viral/bacterial antigens, rather than by the SLA/LP antigen itself [16]. Nevertheless, previous attempts to look for evidence of cross-reactivity of the immunodominant region that is specifically recognized by SLA/LP autoantibodies with microbial antigens have been unsuccessful [16], 
leading to the conclusion that SLA/LP autoimmunity is autoantigen-driven, rather than being driven by molecular mimicry [18].

The present study suggests that local sequence similarity between SLA/LP and a non-homologous bacterial protein from Rickettsia spp. can drive autoimmunity to SLA/LP, through initial $\mathrm{CD} 4^{+} \mathrm{T}$ cell recognition and subsequent humoral response.

\section{Methods}

\section{Sequence analysis}

Residues encompassed by positions 450-495 of SLA/LP autoantigen (O-phosphoserine (Sep)-tRNA:selenocysteine (Sec)-tRNA synthase) from Homo sapiens [UniProt:Q9HD40, sequence variant AAD33963.2 according to [4]] were used as query in the non-redundant (nr) protein sequence database (GenBank CDS translations [19], PDB [20] and UniProtKB/ Swiss-Prot [21]) search, by means of the BLAST server [22]. Algorithm parameters were kept at their default values except for word size, which was set to 2 to enhance search sensibility. Sequence display and alignment relied on the program Jalview [23]. Secondary structure and solvent accessibility were predicted with JPred3 [24]. Predictions of intrinsically disordered regions (IDRs) were carried out as described previously [25].

\section{Modeling of the interaction HLA-DRB1*03:01-peptide}

The crystal structure of HLA-DRB1*03:01 [UniProt:P01912] in complex with a 15 residues fragment (87-101) of invariant chain called CLIP was obtained by PDB [PDB:1A6A; [26]]. The CLIP fragment was subsequently used as structural template to model the potential conformation of other interacting peptides. Molecular modeling relied on the program Molecular Operating Environment [27] and the PyMod tool [28]. In silico mutagenesis of the side-chains of the CLIP fragment was performed to obtain the initial complex between HLA-DRB1*03:01 and the target peptide. Then, after manual adjustment, energy minimization was performed on the whole system. Initially, to allow added hydrogens to adjust to the crystallographically defined environment, the position of the heavy atoms of the binary complex were fixed, and steepest descents steps of energy minimization were performed until the Root Mean Square gradient fell below the $0.05 \AA$ default threshold. Next, while mainchain atoms were fixed, sidechains of every residue comprised in a sphere of $20 \AA$ from the docked peptides were subjected to a gradually decreasing tethering force (from 1000 $\mathrm{Kcal} \cdot \AA^{-2}$ to $10 \mathrm{Kcal} \cdot \AA^{-2}$ ) using again steepest descents, until the Root Mean Square gradient fell below the default threshold. Finally, a decreasing tethering force (until the system was totally relaxed) was applied on every atom comprised in a sphere of $10 \AA$ from the docked peptides, using conjugated gradients, until the maximum gradient was less than $0.0001 \AA$. The Amber99 forcefield, a distancedependent dielectric constant and a cut-off distance of $40 \AA$ were used during each simulation.

An approximate estimate of the binding free energy for both complexes was computed by means of the FastContact 2.0 server [29]. The algorithm implemented in FastContact is based on a statistically determined desolvation contact potential and Coulomb electrostatics, and reports residue contact free energies that rapidly highlight the hotspots of the interaction. 
Surface electrostatic calculations were performed by using the Adaptive PoissonBoltzmann Solver (APBS) software [30].

\section{Results}

\section{Sequence similarity and structure analysis}

Databank searches identified a set of 120 KDa surface antigens from Rickettsia prowazekii (for example, PS 120 protein, UniProt:Q9ZD49), sharing a region of high (Score = 29.6 bits; Identities = 16/29 (55\%); Positives = 19/29 (66\%), Gaps = 0/29 (0\%)) and significant $\left(\mathrm{E}\right.$-value $\left.=2 e^{-04}\right)$ similarity with residues $451-479$ of SLA/LP (Figure 1). The identified region of PS 120 protein spans residues 789-817 of Q9ZD49, which are evolutionarily well conserved amongst other strains and members of the Rickettsiales. In particular, the sequence segment DDIYNKTQDV at positions 808-817 (Figure 1) is almost invariant (see Additional file 1). Interestingly, this sequence is also conserved in SLA/LP antigen where it has been shown to be essential for immune response [16].

The observed sequence similarity between the SLA/LP immunodominant antigen and the sequence 789-817 of PS $120 \mathrm{kDa}$ protein from $R$. prowazekii suggests that these polypeptide regions share a similar secondary structure context. To investigate this issue, secondary structure, accessibility and IDRs predictions were carried out on the complete sequences of SLA/LP and PS 120 protein. The results suggest the presence, for the immunodominant polypeptide region (451-490), of a helix-loop-helix secondary structure element that is accessible to the solvent. The element (helix-loop-helix) is predicted for the corresponding polypeptide region of PS 120 protein (Figure 1).

Simulation of the interaction between HLA-DRB1*03:01 and the peptides SLA/LP $452-465$, and PS $120_{790-804 .}$

$\mathrm{CD}^{+} \mathrm{T}$ cells are the main effectors in most autoimmune diseases. Thus, molecular mimicry depends on demonstrating that these $\mathrm{T}$ cells can be activated by antigenic determinants of an infectious agent that are similar to the determinants present in the host [31]. A recent study on auto-antigenic SLA/LP peptides targeted by $\mathrm{CD} 4^{+} \mathrm{T}$ cells, pinpointed residues 452-465 (hereinafter dubbed peptide "A", sequence NRLDRCLKAVRKER) of the C-terminal, immunodominant region of SLA/LP, as the optimal epitope for CD4 ${ }^{+} \mathrm{T}$ cells expressing the AIH disease susceptibility gene HLA-DRB1*03:01 [15]. As shown in Figure 1,




the peptide "A" shares high sequence similarity to the sequence QNLDRELKAQNINE encompassed by positions 790-803 of the PS 120 from Rickettsia prowazekii, (hereinafter called peptide "B"). However, since it is well known that sequence similarity alone is not sufficient for mimicry in autoimmune diseases, we decided to model the interaction of HLA-DRB1"03:01 with peptide "A", whose interaction has been already proven, and to compare it with a hypothetical complex between HLA-DRB1*03:01 and peptide "B" (Figure 2). Residue Leu 454 of peptide "A" (corresponding to Leu 792 of peptide "B") was chosen as hydrophobic anchoring sites at the P1 pocket, since: 1) it is well known that filling this pocket with a hydrophobic residue constitutes a primary requirement for epitope binding to HLA and epitope selection [32,33]; 2) the P1 pocket of HLA-DRB1*03:01 is located at the beginning of the peptide binding groove, therefore the hydrophobic anchor site should be located at the $\mathrm{N}$-terminus or C-terminus of an interacting peptide, and Leu 454 is the only hydrophobic residue of peptide " $\mathrm{A}$ " fulfilling this requirement. Following the choice of the P1 pocket anchoring site, the main-chain of peptide " $\mathrm{A}$ " and peptide " $\mathrm{B}$ " were initially modeled by assigning the coordinates of the CLIP fragment. The rationale of this procedure is that all of the peptides binding to MHC class II adopt a similar type II polyproline helix conformation, as they interact with the binding groove [34]. In silico mutagenesis of the side-chains of the CLIP fragment was then performed to obtain initial HLA-DRB1*03:01-peptide "A" and HLA-DRB1"03:01-peptide "B" complexes. The sidechain rotamers of peptide "A" and peptide "B" were chosen in order to keep the most similar orientation of the corresponding CLIP peptide, and to interact with the same

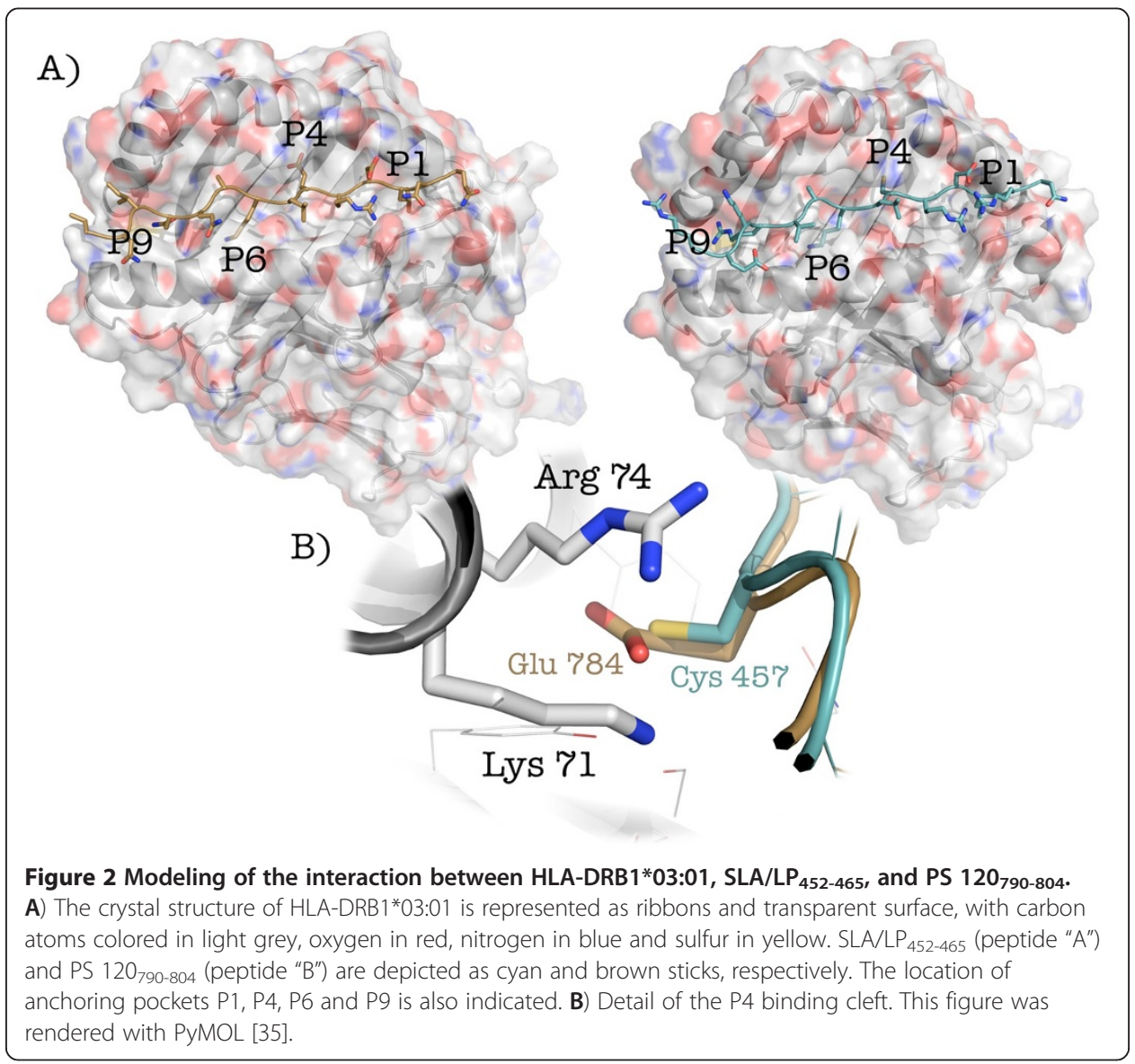


functional residues. Particular care was taken to remove the steric clashes in the resulting complex.

After manual docking and energy minimization of the complexes, peptide "A" and "B" showed good predicted binding affinities for HLA-DRB1*03:01 (-33.6 Kcal/mol for peptide "A" and $-28.1 \mathrm{Kcal} / \mathrm{mol}$ for peptide "B"). Both peptides twist in the typical type II polyproline helix, with the sequestration of peptide side chains in polymorphic P1, P4, P6, and P9 pockets in the HLA protein, which have been identified as major anchors [33]. Table 1 reports the receptor-ligand residue free energy contacts for HLA-DRB1*03:01-peptide "A" and HLA-DRB1"03:01-peptide "B" complexes. As expected, residue Leu 454 of peptide "A" (Leu 792 of peptide "B") fits well in the highly hydrophobic P1 pocket, formed by residues Phe 24, Ile 31, Phe 32, Trp 43, Ala 52, Asn 82, Val 85, Val 86 and Phe 89 of HLA-DRB1*03:01 (predicted desolvation free energy: $-5.624 \mathrm{Kcal} / \mathrm{mol}$ and -5.440 for peptide "A" and "B", respectively). According to Table 1, a "hot spot" of interaction between HLA-DRB1"03:01 and peptide " $\mathrm{B}$ " is located at the P4 binding pocket, and is represented by the salt bridges involving Lys at position 71 and Arg at position 74 of the HLA protein, and Glu 795 of PS120 peptide (Lys 71-Glu 795, -5.191 Kcal/mol; Arg 74-Glu 795, -3.153 $\mathrm{Kcal} / \mathrm{mol})$. These interactions are not present in SLA/LP immunodominant peptide, where the Glu residue is substituted for Cys 457. The P6 binding pocket of HLA-DRB1* 03:01 is negatively charged, being occupied by residues Glu 9 of chain A, Glu 11 and Asp 66 of chain B. In this pocket are accommodated the positively charged residue Lys 459

\begin{tabular}{|c|c|c|}
\hline HLA-DRB1*03:01 & SLA/LP & Free energy (Kcal/mol) \\
\hline Asp B57 & $\operatorname{Arg} 462$ & -7.382 \\
\hline Glu A55 & $\operatorname{Arg} 453$ & -5.015 \\
\hline Asp A66 & Lys 459 & -4.108 \\
\hline Glu B9 & Lys 549 & -2.518 \\
\hline Arg A76 & Arg 465 & -2.381 \\
\hline Leu B53 & Arg 462 & -2.013 \\
\hline Ser B11 & Lys 459 & -1.879 \\
\hline Tyr B78 & Cys 457 & -1.158 \\
\hline Val B85 & Leu 454 & -1.130 \\
\hline Phe A54 & Leu 454 & -1.081 \\
\hline HLA-DRB1*03:01 & PS 120 & Free energy (Kcal/mol) \\
\hline Lys B71 & Glu 795 & -5.191 \\
\hline Asp A66 & Lys 797 & -3.279 \\
\hline Arg B74 & Glu 795 & -3.153 \\
\hline Glu B9 & Lys 797 & -2.671 \\
\hline Arg B74 & Leu 796 & -1.972 \\
\hline Asp B57 & Asn 800 & -1.425 \\
\hline Tyr B61 & Ile 801 & -1.235 \\
\hline Glu A55 & Arg 794 & -1.211 \\
\hline Phe A54 & Leu 792 & -1.121 \\
\hline Val B85 & Leu 792 & -1.057 \\
\hline
\end{tabular}


and Lys 797 of peptides "A" and "B", respectively, which favourably contribute to the free energy of binding $(-3.609 \mathrm{Kcal} / \mathrm{mol}$ and $-4.072 \mathrm{Kcal} / \mathrm{mol}$, respectively). Finally, the positively charged side-chain of Arg 462 of peptide " $\mathrm{A}$ " is located inside the P9 binding pocket, where it interacts with Asp 57 (chain B) of HLA-DRB1*03:01. The latter represents the most favourable interaction between HLA-DRB1*03:01 and peptide "A", according to residue free energy contacts $(-7.382 \mathrm{Kcal} / \mathrm{mol})$. In case of peptide "B", Arg 462 is replaced by Asn 800, which is hydrogen-bonded to Asn 69 (chain A) and Asp 57 (chain B) of HLA-DRB1*03:01.

\section{Discussion}

PLP-enzymes are involved in a number of diseases, including autoimmunity [36-38], and Bioinformatics approaches have been extensively exploited in the past to understand the molecular basis of human disorders involving PLP-enzymes [39,40], and to design drugs specifically targeting such enzymes [41,42].

This study suggests the possible role of molecular mimicry between microbial antigens and the immunodominant region of a PLP dependent enzyme, SLA/LP. The hypothesis that foreign antigens of bacterial proteins sharing homology with the SLA/LP protein might drive autoimmunity, via a molecular mimicry mechanism, has been already debated [16]. By performing sequence similarity searches in publicly available databases, these authors identified the protein MJ0610 from the Archaea Methanococcus jannaschii [GenBank:U67509], a non-pathogenic, hyperthermophillic organism, as the only bacterial candidate for molecular mimicry to occur. Subsequent attempts to look for evidence of cross-reactivity of the immunodominant region that is specifically recognized by SLA/LP autoantibodies with MJ0610 have been unsuccessful [16]. The huge amount of sequence information that is available nowadays in protein databases prompted us to search again for bacterial proteins that could be able to trigger an autoimmune response in $\mathrm{AIH}$, on the base of a molecular mimicry event. The obtained results suggest that a highly significant, local sequence similarity between SLA/LP and a non-homologous bacterial protein from Rickettsia spp. might drive autoimmunity to SLA/LP, through initial CD4 $4^{+} \mathrm{T}$ cell recognition and subsequent humoral response.

The PS $120 \mathrm{kDa}$ protein from $R$. prowazekii is a 1022 residues sequence of unknown function, belonging to the family of $120 \mathrm{KDa}$ Rickettsia surface antigens [Pfam: 12574] [43], which may be used as antigens for immune response against the Rickettsia species [44]. This protein is a close homolog of R. conorii PS $120 \mathrm{kDa}$ protein [UniProt:Q52658; identities $=640 / 1019(63 \%)$ ], an antigen that is recognized by antirickettsial antibodies in sera from humans infected with spotted fever group rickettsiae, and that is supposed to be an important stimulator of the host immune response [44].

Together with mono-dimensional amino acid sequence similarity, the three-dimensional conformational fit of the immunodominant epitope from host with a polypeptide chain from the pathogen is also a key aspect in for molecular mimicry to occur [45]. Since SLA/LP autoantibodies react preferentially with conformational epitopes [46], we considered the possibility that, beside sequence similarity, the SLA/LP immunodominant antigen and residues 789-817 of PS $120 \mathrm{kDa}$ protein from Ricketsia spp. could present a similar secondary structure motif and solvent accessibility. The crystal structure of the human SepSecS-tRNA ${ }^{\text {Sec }}$ binary complex [PDB: 3HL2] revealed that the first 14 residues 
(450-463) of the immunodominant region of SLA/LP adopt an $\alpha$-helix secondary structure, while the remaining residues (464-501) showed a disordered state and were not solved in any of the crystal structures of human SepSecS already determined [47-49]. Our structural analysis on the immunodominant region of SLA/LP agrees with previous bioinformatics studies [50], suggesting that residues 450-490 of SLA/LP fold in a helixloop-helix conformation that is accessible to the solvent, and therefore easily recognized by SLA/LP autoantibodies. Moreover, we suggest that the corresponding region of PS 120 KDa protein could adopt a similar conformation and solvent exposure, which therefore would render it amenable to the proteolytic cleavage that is necessary for the immune response to take place.

The recent findings that, of two immunodominant T cell peptides of the SLA/LP protein, one overlaps with the immunodominant region that is recognized by SLA/LP autoantibodies [15], provide an interesting link between humoral and cell-mediated immune response in $\mathrm{AIH}$, and prompted us to investigate further this issue, by modeling the interaction between the AIH susceptibility allele HLA-DRB1*03:01, human SLA/LP $452-465$, and the corresponding PS $120_{790-802}$ from $R$. prowazekii. The obtained complexes predict for both peptides a similar binding mode and affinity, suggesting that $\mathrm{CD} 4^{+} \mathrm{T}$ cells recognizing self-antigens in the context of the alleles HLA-DRB1*03:01 could indeed cross-react with foreign Rickettsia spp. peptides. Most importantly, a "hot spot" of interaction between HLA-DRB1"03:01 and peptide "B" is represented by the salt bridges involving Lys at position 71 and Arg at position 74 of the HLA protein, and Glu 795 of PS120 peptide. This observation could potentially provide a mechanistic explanation to the fact that a positively charged residue at position 71 has been indicated as critical for AIH susceptibility in all of the HLA alleles identified to date [14]. Indeed, the Lys 71-Glu 795 interaction could be essential for HLA-antigen recognition and binding, and the lack of this interaction could prevent HLA alleles from binding the foreign peptides and triggering the molecular mimicry event. Indeed, in the DRB1*1501 allele, which confers resistance to AIH [51], Lys 71 is replaced by an Ala residue. Moreover, beside Lys 71, the presence of Arg at position 74 is also related to an increased susceptibility to AIH [51]. Interestingly, in type 1 diabetes mellitus, where molecular mimicry has been proposed as an explanation of the pathogenesis of the disease, a similar mechanism has been observed: an aspartate residue at position 57 of the DQb- $\beta$ polypeptide chain confers protection, whereas a serine is associated with an increased risk of disease [52].

Rickettsia is a genus of Gram-negative, obligate intracellular parasites, which are carried by many ticks, fleas, and lice, and cause diseases in humans such as typhus, rickettsialpox, Boutonneuse fever, African tick bite fever, Rocky Mountain spotted fever, Flinders Island spotted fever and Queensland tick typhus (Australian Tick Typhus) [53]. Actually, rickettsial microorganisms are not necessarily pathogenic species, and are indeed ubiquitous in human populations, where they normally live in peaceful coexistence with the human beings [54]. These organisms represent one of the closest living relatives to bacteria that originated the mitochondria inside most eukaryotic cells [55]. Rickettsiales display small, degraded genomes, with a high propensity of genetic exchange occurring between bacteria that infect the same host and with the eukaryotic hosts themselves. Crosstalk between host and bacteria appears to be mediated by proteins containing motifs with high similarity to eukaryotic-like repeats [53]. The widespread presence of rickettsial microorganisms amongst humans, the bacteria-host genetic exchange and the local similarity of 
eukaryotic-like repeats, led in the past to the hypothesis that rickettsiales are indeed related to the emergence of human autoimmune diseases, e.g., multiple sclerosis [54].

\section{Conclusions}

Diagnosis of AIH is usually based on a number of outcomes from clinical, laboratory, and histological exams. Anti-SLA/LP autoantibodies are considered highly specific markers of AIH [2], and SLA/LP autoantibodies recognize the SLA/LP antigen with high sensitivity and specificity [4]. On the contrary, commonly detected autoantibodies such as antinuclear and smooth muscle antibodies are not specific for the disease [3,4]. The presence of different autoantibodies, that is definitively an important part of the final diagnosis, reflects the complex and diverse interplay between environmental triggering factors, autoantigens and immunogenetic predisposition of the individual.

Though it is highly unlikely that any single infectious agent would be exclusively associated with the disease, molecular mimicry provides an elegant framework as to how cross-reactivity between antigens from a foreign agent with self-proteins may trigger such disease.

The predicted sequence and structural similarity between the immunodominant epitopes of the SLA/LP antigen and PS 120 protein from Rickettsia spp. could account for crossrecognition to occur in autoimmune hepatitis, and contribute to the development of this disease. The presented findings are supported by coherent and rigorous theoretical considerations and form the basis of a well-grounded hypothesis that can be experimentally tested. Obviously, $\mathrm{CD}^{+} \mathrm{T}$ cell recognition requires that antigen is degraded by $\mathrm{pH}$-dependent proteolysis in phago-endosomal compartments of APCs. This means that only a portion of peptides are efficiently generated from a given antigen and result available to be loaded onto MHC class II molecules. Several cryptic peptides might not have in vivo relevance, despite being potential MHC class II binders, if they are not generated during proteolysis of the entire protein.

To conclude that the suggested peptides are authentic immunodominant T-cell epitopes, future studies should be aimed at detecting also the frequency of memory B and T cells specific to PS 120 protein epitope in AIH patients and healthy donors. If such studies will be confirmed, they may contribute to open new perspectives for AIH prevention and therapy.

\section{Additional file}

Additional file 1: Sequence alignment of PS 120 protein (region 789-817) from different Rickettsiales. Numbering refers to $R$. prowazekii.

Competing interests
The authors declare that they have no competing interests.

Authors' contributions

AP carried out structural analysis, helped in sequence analysis and participated in drafting the manuscript. SP carried out sequence analysis, conceived the study and participated in drafting the manuscript. Both authors read and approved the final manuscript. funded by the Italian Ministero dell'Istruzione, Università, Ricerca (prot. C26A11S2S3). 
References

1. Krawitt EL: Autoimmune hepatitis. N Engl J Med 1996, 334:897-903.

2. Manns M, Gerken G, Kyriatsoulis A, Staritz M, Meyer zum Büschenfelde KH: Characterisation of a new subgroup of autoimmune chronic active hepatitis by autoantibodies against a soluble liver antigen. Lancet 1987, 7:292-294

3. Kanzler S, Weidemann C, Gerken G, Löhr HF, Galle PR, Meyer zum Büschenfelde KH, Lohse AW: Clinical significance of autoantibodies to soluble liver antigen in autoimmune hepatitis. J Hepatol 1999, 31:635-640.

4. Wies I, Brunner S, Henninger J, Herkel J, Kanzler S, Meyer zum Büschenfelde KH, Lohse AW: Identification of target antigen for SLA/LP autoantibodies in autoimmune hepatitis. Lancet 2000, 355:1510-1515.

5. Gelpi C, Sontheimer EJ, Rodriguez-Sanchez JL: Autoantibodies against a serine tRNA-protein complex implicated in cotranslational selenocysteine insertion. Proc Natl Acad Sci U S A 1992, 89:9739-9743.

6. Paiardini A, Bossa F, Pascarella S: Evolutionarily conserved regions and hydrophobic contacts at the superfamily level: The case of the fold-type I pyridoxal-5'-phosphate-dependent enzymes. Protein Sci 2004, 13:2992-3005

7. Singh R, Spyrakis F, Cozzini P, Paiardini A, Pascarella S, Mozzarelli A: Chemogenomics of pyridoxal 5'-phosphate dependent enzymes. J Enzyme Inhib Med Chem 2013, 28:183-194.

8. Cellini B, Bertoldi M, Montioli R, Paiardini A, Borri Voltattorni C: Human wild-type alanine:glyoxylate aminotransferase and its naturally occurring G82E variant: functional properties and physiological implications. Biochem J 2007, 408:39-50.

9. di Salvo ML, Florio R, Paiardini A, Vivoli M, D’Aguanno S, Contestabile R: Alanine racemase from Tolypocladium inflatum: a key PLP-dependent enzyme in cyclosporin biosynthesis and a model of catalytic promiscuity. Arch Biochem Biophys 2013, 529:55-65.

10. Yuan J, Palioura S, Salazar JC, Su D, O'Donoghue P, Hohn MJ, Cardoso AM, Whitman WB, Söll D: RNA-dependent conversion of phosphoserine forms selenocysteine in eukaryotes and archaea. Proc Natl Acad Sci U S A 2006, 103:18923-18927.

11. Czaja AJ: Autoimmune hepatitis-approach to diagnosis. MedGenMed 2006, 23:55

12. Czaja AJ, Strettell MD, Thomson LJ, Santrach PJ, Moore SB, Donaldson PT, Williams R: Associations between alleles of the major histocompatibility complex and type 1 autoimmune hepatitis. Hepatology 1997, 25:317-323.

13. McFarlane IG: Definition and classification of autoimmune hepatitis. Semin Liver Dis 2002, 22:317-324

14. Czaja AJ, Donaldson PT: Genetic susceptibilities for immune expression and liver cell injury in autoimmune hepatitis. Immunol Rev 2000, 174:250-259.

15. Mix H, Weiler-Normann C, Thimme R, Ahlenstiel G, Shin EC, Herkel J, David CS, Lohse AW, Rehermann B: Identification of CD4 T-cell epitopes in soluble liver antigen/liver pancreas autoantigen in autoimmune hepatitis. Gastroenterology 2008, 135:2107-2118.

16. Herkel J, Heidrich B, Nieraad N, Wies I, Rother M, Lohse AW: Fine specificity of autoantibodies to soluble liver antigen and liver/pancreas. Hepatology 2002, 35:403-408.

17. Lunardi C, Bason C, Navone R, Millo E, Damonte G, Corrocher R, Puccetti A: Systemic sclerosis immunoglobulin $\mathrm{G}$ autoantibodies bind the human cytomegalovirus late protein UL94 and induce apoptosis in human endothelial cells. Nat Med 2000, 6:1183-1186.

18. Wang CX, Teufel A, Cheruti U, Grötzinger J, Galle PR, Lohse AW, Herkel J: Characterization of human gene encoding SLA/LP autoantigen and its conserved homologs in mouse fish fly and worm. World J Gastroenterol 2006, 12:902-907.

19. Benson DA, Karsch-Mizrachi I, Lipman DJ, Ostell J, Sayers EW: GenBank. Nucleic Acids Res 2011, 39:D32-D37.

20. Berman HM, Kleywegt GJ, Nakamura H, Markley JL: The Protein Data Bank at 40: reflecting on the past to prepare for the future. Structure 2012, 20:391-396.

21. Consortium UP: Reorganizing the protein space at the Universal Protein Resource (UniProt). Nucleic Acids Res 2012, 40:D71-D75

22. Altschul SF, Madden TL, Schäffer AA, Zhang J, Zhang Z, Miller W, Lipman DJ: Gapped BLAST and PSI-BLAST: a new generation of protein database search programs. Nucleic Acids Res 1997, 25:3389-3402.

23. Waterhouse AM, Procter JB, Martin DM, Clamp M, Barton GJ: Jalview Version 2-a multiple sequence alignment editor and analysis workbench. Bioinformatics 2009, 25:1189-1191.

24. Cole C, Barber JD, Barton GJ: The Jpred 3 secondary structure prediction server. Nucleic Acids Res 2008, 36:W197-W201.

25. Paiardini A, Caputo V: Insights into the interaction of sortilin with proneurotrophins: a computational approach. Neuropeptides 2008, 42:205-214

26. Ghosh $\mathrm{P}$, Amaya M, Mellins E, Wiley DC: The structure of an intermediate in class II MHC maturation: CLIP bound to HLA-DR3. Nature 1995, 378:457-462.

27. MOE Molecular Operating Environment. http://www.chemcomp.com/MOE-Molecular_Operating Environment.htm.

28. Bramucci E, Paiardini A, Bossa F, Pascarella S: PyMod: sequence similarity searches multiple sequence-structure alignments and homology modeling within PyMOL. BMC Bioinformatics 2012, 28:S2.

29. Camacho CJ, Zhang C: FastContact: rapid estimate of contact and binding free energies. Bioinformatics 2005, 21:2534-2536.

30. Baker NA, Sept D, Joseph S, Holst MJ, McCammon JA: Electrostatics of nanosystems: application to microtubules and the ribosome. Proc Natl Acad Sci USA 2001, 98:10037-10041.

31. Albert LJ, Inman RD: Molecular mimicry and autoimmunity. N Engl J Med 1999, 341:2068-2074.

32. Natarajan SK, Stern LJ, Sadegh-Nasseri S: Sodium dodecyl sulfate stability of HLA-DR1 complexes correlates with burial of hydrophobic residues in pocket 1. J Immunol 1999, 162:3463-3470.

33. Ferrante A, Gorski J: Cooperativity of hydrophobic anchor interactions: evidence for epitope selection by MHC class II as a folding process. J Immunol 2007, 178:7181-7189. 
34. Jardetzky TS, Brown JH, Gorga JC, Stern $\sqcup$, Urban RG, Strominger JL, Wiley DC: Crystallographic analysis of endogenous peptides associated with HLA-DR1 suggests a common polyproline II-like conformation for bound peptides. Proc Natl Acad Sci U S A 1996, 93:734-738.

35. The PyMOL Molecular Graphics System. http://www.pymol.org/.

36. Candeloro P, Voltattorni CB, Perniola R, Bertoldi M, Betterle C, Mannelli M, Giordano R, De Bellis A, Tiberti C, Laureti $\mathrm{S}$, Santeusanio F, Falorni A: Mapping of human autoantibody epitopes on aromatic L-amino acid decarboxylase. J Clin Endocrinol Metab 2007, 92:1096-1105.

37. di Salvo ML, Contestabile R, Paiardini A, Maras B: Glycine consumption and mitochondrial serine hydroxymethyltransferase in cancer cells: The heme connection. Med Hypotheses 2013, 80:633-636.

38. Giardina G, Montioli R, Gianni S, Cellini B, Paiardini A, Voltattorni CB, Cutruzzolà F: Open conformation of human DOPA decarboxylase reveals the mechanism of PLP addition to Group II decarboxylases. Proc Natl Acad Sci U S A 2011, 108:20514-20519.

39. Montioli R, Oppici E, Cellini B, Roncador A, Dindo M, Voltattorni CB: S250F variant associated with aromatic amino acid decarboxylase deficiency: molecular defects and intracellular rescue by pyridoxine. Hum $\mathrm{Mol}$ Genet 2013, 22:1615-1624.

40. Pittman AM, Lage MD, Poltoratsky V, Vrana JD, Paiardini A, Roncador A, Cellini B, Hughes RM, Tucker CL: Rapid profiling of disease alleles using a tunable reporter of protein misfolding. Genetics 2012, 192:831-842.

41. Daidone F, Montioli R, Paiardini A, Cellini B, Macchiarulo A, Giardina G, Bossa F, Borri Voltattorni C: Identification by virtual screening and in vitro testing of human DOPA decarboxylase inhibitors. PLoS One 2012, 7:e31610.

42. Daidone F, Florio R, Rinaldo S, Contestabile R, di Salvo ML, Cutruzzolà F, Bossa F, Paiardini A: In silico and in vitro validation of serine hydroxymethyltransferase as a chemotherapeutic target of the antifolate drug pemetrexed. Eur J Med Chem 2011, 46:1616-1621.

43. Punta M, Coggill PC, Eberhardt RY, Mistry J, Tate J, Boursnell C, Pang N, Forslund K, Ceric G, Clements J, Heger A, Holm L, Sonnhammer EL, Eddy SR, Bateman A, Finn RD: The Pfam protein families database. Nucleic Acids Res 2012, 40:D290-D301.

44. Schuenke KW, Walker DH: Cloning sequencing and expression of the gene coding for an antigenic 120kilodalton protein of Rickettsia conorii. Infect Immun 1994, 162:904-909.

45. Kohm AP, Fuller KG, Miller SD: Mimicking the way to autoimmunity: an evolving theory of sequence and structural homology. Trends Microbiol 2003, 11:101-105.

46. Ma Y, Okamoto M, Thomas MG, Bogdanos DP, Lopes AR, Portmann B, Underhill J, Dürr R, Mieli-Vergani G, Vergan D: Antibodies to conformational epitopes of soluble liver antigen define a severe form of autoimmune liver disease. Hepatology 2002, 35:658-664.

47. Araiso Y, Palioura S, Ishitani R, Sherrer RL, O'Donoghue P, Yuan J, Oshikane H, Domae N, Defranco J, Söll D, Nurek O: Structural insights into RNA-dependent eukaryal and archaeal selenocysteine formation. Nucleic Acids Res 2008, 36:1187-1199

48. Ganichkin OM, Xu XM, Carlson BA, Mix H, Hatfield DL, Gladyshev VN, Wahl MC: Structure and catalytic mechanism of eukaryotic selenocysteine synthase. J Biol Chem 2008, 283:5849-5865.

49. Palioura S, Sherrer RL, Steitz TA, Söll D, Simonovic M: The human SepSecS-tRNASec complex reveals the mechanism of selenocysteine formation. Science 2009, 325:321-325.

50. Kernebeck T, Lohse AW, Grötzinger J: A bioinformatical approach suggests the function of the autoimmune hepatitis target antigen soluble liver antigen/liver pancreas. Hepatology 2001, 34:230-233.

51. Strettell MD, Donaldson PT, Thomson LJ, Santrach PJ, Moore SB, Czaja AJ, Williams R: Allelic basis for HLA-encoded susceptibility to type 1 autoimmune hepatitis. Gastroenterology 1997, 112:2028-2035.

52. Todd JA, Bell JI, McDevitt HO: HLA-DQ beta gene contributes to susceptibility and resistance to insulin-dependent diabetes mellitus. Nature 1987, 329:599-604.

53. Renvoisé A, Merhej V, Georgiades K, Raoult D: Intracellular Rickettsiales Insights into manipulators of eukaryotic cells. Trends Mol Med 2011, 17:573-583.

54. Frykholm BO: On the question of infectious aetiologies for multiple sclerosis schizophrenia and the chronic fatigue syndrome and their treatment with antibiotics. Med Hypotheses 2009, 72:736-739.

55. Emelyanov W: Rickettsiaceae rickettsia-like endosymbionts and the origin of mitochondria. Biosci Rep 2001, 21:1-17.

doi:10.1186/1742-4682-10-25

Cite this article as: Paiardini and Pascarella: Structural mimicry between SLA/LP and Rickettsia surface antigens as a driver of autoimmune hepatitis: insights from an in silico study. Theoretical Biology and Medical Modelling 2013 10:25. 